\title{
Modeling and Portfolio Optimization of Stochastic Discrete-Event System through Markovian Approximation: an Open-Pit Mine Study
}

\author{
Roberto G. Ribeiro $^{1}$ Rodney R. Saldanha ${ }^{2}$ Carlos A. Maia ${ }^{2}$ \\ ${ }^{1}$ Graduate Program in Electrical Engineering, Federal University of Minas Gerais - UFMG, Brazil, \\ rogoribegmail.com \\ ${ }^{2}$ Dep. of Electrical Engineering, Federal University of Minas Gerais - UFMG, Brazil, \\ \{rodney, maia\} @ cpdee.ufmg.br
}

\begin{abstract}
Operation in an open-pit mining is a complex task with stochastic nature. Usually, this kind of system is analyzed by means of DES (Discrete Event System) simulation. This work considers optimizing the investment in new projects in such a way to reach maximum production of an open-pit mine. When a DES model is associated with an optimization problem, the time taken to run such model is a crucial aspect. In order to analyze the project impacts in a reasonable time, this work presents a DES markovian model which represents a load-haulage cycle. The results obtained were compared with the results acquired from validated simulation models which represent the same system. In the optimization context, the complexity is exponential. Therefore, this work proposes a formulation that considers the inter-relationship between projects, which aims to help decision makers. Instead of trying all the possible projects combinations, the proposed method searches for identifying the set of projects that produce good feasible solutions based on performance measure from designed DES model.
\end{abstract}

Keywords: Markov chain, closed queuing network, project portfolio, open-pit mining.

\section{Introduction}

Typically, Project Portfolio Optimization decisions are strategic decisions that are made on a yearly basis. For open-pit mine, investments in its operations are needed to reduce the production cost, enhance production, eliminate bottlenecks and to improve the use of system resources. Thus, in a Brazilian mining, several projects are proposed annually in order to improve the company competitiveness. The Project Portfolio consists of many actions, including road improvements, truck or crane acquisition, hire operator, etc. Each project impacts in a specific indicator of the open-pit mine. A preliminary goal is to identify how these projects influence the company competitiveness. A fundamental goal of any mining project is maximizing the total mine ore production in a specific time horizon. Nevertheless, to improve some indicator cannot result in an effective contribution for this goal.

The open-pit-mine is a complex and stochastic system, in which interactions between several agents impact heavily on the total mine ore production. Usually, this kind of system is analyzed by means of DES simulation. According to (Banks, 2000), simulation is the imitation of the operation of a real-word process or system over time, involving the generation of an artificial history of the system. In order to analyze the real impact of each project, a DES simulation model can be used. Therefore, it is possible to estimate and make better decisions.

When DES simulation model is associated with a discrete decision problem, we can associate the control variables to a discrete optimization problem. Discrete optimization with simulation is a methodology known in the literature as discrete optimization via simulation. According to (Nelson, 2010), this methodology addresses solving problems with a countable number of feasible solutions, when the system is complex enough that its expected value is estimated by running simulation.

Most commercial DES simulation software are associated with some optimization software. However, these softwares use meta-heuristics or heuristics that, although efficient in many cases and modeled to be generic and applicable in various contexts, it does not exploit the problem features and hence tend to be less efficient. Fu (Fu, 2002) summarizes the optimization packages used by most popular commercial DES simulation softwares and the search strategy used as well. According to (Fu, 2002), algorithms that apply a very general way often have a slow convergence in practice.

In a portfolio optimization, the goal is to determine a set of projects that maximizes some indicator, such as total mine production index. Each portfolio is formed at least by one project and correct projects combinations can maximize the expected return. From the optimization point of view, the number of combinations has an exponential growth. Thus, usually it is not possible analyze all the possibilities, especially when each evaluation is obtained using DES simulation, since this methodology can be very burden. 
According to (Bolch et al., 1998), the main drawback of DES simulation models is the time taken to run such models for large and realistic systems particularly when results with high accuracy are desired. Thereby, combining DES simulation with optimization is a major challenge.

Taken into account the difficulties inherent to discrete optimization via simulation, two approaches to research can be explored. The first consist in reducing the computational time used in the function evaluation. When we consider a DES simulation model as an objective function of an optimization problem, the time taken to run such model is a crucial aspect. The second approach consist in developing a specific optimization method to the problem that explores peculiarities of the modeled system.

\subsection{First step - DES model}

The first step aims to create a modeling of stochastic DES through markovian approximation. This strategy was motivated by successful application in the field of the performance evaluation of computational system. According to (Bolch et al., 1998), a cost-effective alternative to DES simulation consists of analytic models, which can provide relatively quick answers and more insight to the system being studied. Before the creation of nowadays computational technology, problems with stochastic nature were generally solved analytically. Marie (Marie, 2011) claims that scientific ambition was limited by computing power, i.e., it was necessary to use the imagination to look for approximations in order to reduce a state space to a few hundred states. Recently, (Marie, 2011) observed that huge amounts of available computing resources increase the trend to solve models through simulation and did not encouraged researchers to look for tractable analytical solutions.

It is important to point out that simulation is enshrined as a good methodology to treat DES model. However, in the optimization context, the number of evaluations depends on the time taken by run the designed model. Reference (Ekren et al., 2013) presents an analytical model for an autonomous vehicle storage and retrieval system. The authors model a material-handling system as a semi-open queuing network to be used instead of DES simulation. According to (Ekren et al., 2013), the analytical model is useful in estimating key performance measures of alternate configurations of the system quickly and accurately.

Marie (Marie, 2011) explain that some mathematical/probabilistic properties can be used to analyze problems of stochastic nature without simulation. Therefore, the first step of this study consist to explore and to apply this properties. A load-haulage cycle of a realistic Brazilian open-pit mine is considered. This cycle is modeled as a closed queuing network where all queues (also known as nodes) are connected. The idea is to measure the mean response time of each node, and consequently, the mean cycle time and the total mine production index. Therefore, these performance measures are used in the optimization context to find a good and fast answer.
In this study we consider the cdf (Cumulative Distribution Function) approximation using exponential distribution. We assume that the service time of each node is exponentially distributed while this is not true in the real model. It is important to point out that this kind of approximation cannot provide a reasonable accuracy. Therefore, the results obtained by this approach are compared with the results acquired using validated simulation models presented in (Ribeiro, 2015). These models consider pdf (Probability Density Function) approximation using general distribution. A Petri net model and a SIMAN model representing the same load-haulage cycle are considered. The assumption is to verify whether there are significant difference among the designed analytical approximation model and the mentioned methodologies of DES simulation.

\subsection{Second Step - Portfolio optimization}

The second step of this study consist in determining the set of projects which maximizes the total mine production index respecting the established budget. One approach is to formulate this decision as a knapsack problem which is a classical combinatorial optimization problem (Pisinger, 1994). Figuratively, we can describe this problem as filling a backpack without exceeding a certain volume limit. The decision consists to place in the backpack products that maximize (maximization problem) a specified value, respecting its capacity. In this study, the products are the projects at the portfolio and the capacity is the available budget.

In order to analyze the real impact of witch project, the DES model is used. Hence, it is possible to estimate how much each one increases the total mine production index. Here, we named this estimative as 'gain'. A linear solution can be found maximizing the sum of individual gain. However, we cannot consider each project individually. Due to the open-pit mine features, there are interrelationships among projects. It means that a combination of two or more projects must result in different gain compared with the linear solution. Since it may unfeasible evaluate all projects combinations, the optimization strategy aims to create a formulation where the decision variables set is formed by all individuals projects in the portfolio and all 'relevant combinations'. In this study, a 'relevant combination' is a set with at least two projects with a strong inter-relationship among them. A major challenge is to find how intense this inter-relationship is.

In the load-haulage cycle, the total mine production index depends on the mean response time taken in each node of the cycle. Some projects aims to reduce the service time in a specific node. A disadvantage of applying these projects is the possibility of increasing the mean response time of others nodes. Consequently, neither total cycle time nor total mine production index will be changed. The central core of the optimization method is to use the mean response time of each node to find 'relevant combinations'. The major idea is that this performance measure 
indicates the plant bottlenecks, which must be fixed by the new projects. Section 5 describes the philosophy behind of the strategy.

\section{Stochastic DES model through markovian properties}

A general way to represent a stochastic DES model is through stochastic timed automata. According to (Cassandras, 2008), any timed model for DES requires the specification of a clock structure. This structure is a set of distribution functions, one for each event, characterizing the corresponding lifetimes. Beside that, it determines the dynamic of the system. When all clock sequences are iid (Independent and Identically Distributed) and the interevent times are exponentially distributed, a stochastic DES can be modeled as a Markov Chain (Cassandras, 2008).

Figure 1a shows a stochastic timed automata where $a$ and $d$ are events with occurrence times rates of $\lambda$ and $\mu$, respectively. Considering the markovian properties presented in (Cassandras, 2008), this automata generates a Markov chain illustrated in Figure $1 \mathrm{~b}$.

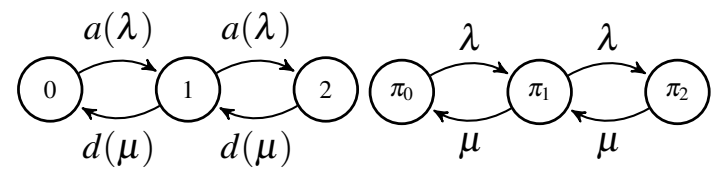

(a) Stoch. timed automata

(b) Markov chain

Figure 1. Stochastic timed automata as Markov Chain

In Figure $1 \mathrm{~b}, \pi_{0}, \pi_{1}$ and $\pi_{2}$ denotes the stationary probability of the states 0,1 and 2, respectively. According to (Cassandras, 2008), a system modeled as a Markov chain is allowed to operate for a sufficiently long period of time so that the state probabilities can reach some fixed values which no longer vary with time. The main objective of using a Markov chain is to compute these probabilities. According to (Bolch et al., 1998), long run dynamics of Markov chains can be studied using a system of linear equations with one equation for each state. Thus, solution of these equations results in stationary probabilities of the Markov chain, consequently, desired performance measures such as mean response time can be easily obtained.

It is known that a DES simulation model can be seen as a parallel composition of many stochastic timed automata. The set of clock structures describes how the DES model evolves as a result of event occurrences over time. Considering that all clock structure of this DES model conforms to the markovian properties, the full DES model can be simplified to a Markov chain (In a parallel composition model, the stationary probabilities are denoted by the $M$-tuple $\pi\left(n_{0}, \ldots, n_{M}\right)$ where $M$ represents the number of individual automata). Accordingly, a system of linear equations to compute the stationary probabilities must still be valid. The problem of this representation is the fact that the cardinality of the state space can grow drastically in a
Markov chain of a complex system. Therefore, computing the stationary probabilities of the states became a hard (even impossible) task.

Fortunately, for a class of Markov Chain, which can be expressed as a of queuing network, very fast numerical solution methods have been developed to derive important performance measures without resorting to the underlying state space (Bolch et al., 1998). One of these methods is known as convolution algorithm. This method is a sufficient analytical technique to obtain the interest measures of this study. This algorithm aims to evaluate an important performance measure without having to compute explicitly the stationary probabilities.

\subsection{Product-Form Networks - Convolution al- gorithm}

Once we approximate the stochastic automata by a Markov Chain and then by a queuing network, to compute the stationary probability it is necessary to consider the theorem proposed by (Jackson, 1957). The Jackson's theorem (also known as product-form) says the steady-state probability of the network can be expressed as the product of the state probabilities of the individual nodes. Based in the Jackson's theorem, (Gordon and Newell, 1967) present a general formulation to compute the stationary probabilities in a closed queuing network. This formulation follows the Eq. 1:

$$
\pi\left(n_{1}, \ldots, n_{M}\right)=\frac{1}{G(N)} \prod_{m=1}^{M} \beta_{m}\left(n_{m}\right)
$$

where $G(N)$ denotes the normalization constant. In this equation, $\beta_{m}\left(n_{m}\right)$ denotes a step function which depends on the number of customer $n_{m}$ in each node $m$. Let $\mu_{m}$ be the service rate of the node $m$, and let $v_{m}$ be the relative arrival rate of the same node, the value of $\beta_{m}\left(n_{m}\right)$ is obtained using Eq. 2.

$$
\beta_{m}\left(n_{m}\right)= \begin{cases}1, & \text { if } n_{m}=0 \\ \frac{\left(\frac{v_{m}}{\mu_{m}}\right)^{n_{m}}}{n_{m}}, & \text { else } \\ \prod_{k=1} b_{m}(k) & \end{cases}
$$

Usually, in a closed queuing network $\mu_{m}$ is known, while $v_{m}$ should be compute. Thus, the balance equations Eq. 3 can be used to compute $v_{m}$, where the parameter $p_{i m}$ denotes the transition probability that a customer departs from node $i$ and arrives at the node $m$.

$$
v_{m}=\sum_{i=1}^{M} v_{j} p_{i m} \quad \forall m=1, \ldots, M .
$$

The step function Eq. 2 depends on the queuing discipline of the node as well. In this study we consider the disciplines: FIFO (First In First Out) and IS (Infinity Server). Thus, $b_{m}(k)$ denotes a step function which depends on the 
queuing discipline and the number of service $q_{m}$, as we can see in Eq. 4.

$$
b_{m}(k)= \begin{cases}0, & \text { if } k=0 \\ \min \left(k, q_{m}\right), & \text { if } 0<k \leq N \quad \& \text { FIFO } \\ k, & \text { if } 0<k \leq N \quad \& \text { IS }\end{cases}
$$

According to Buzen algorithm (Buzen, 1973), the normalization constant $G(N)$ can be computed following Eq. 5 .

$$
g(n, m)= \begin{cases}1, & \text { if } n=0, \forall m \\ \beta_{m}(n), & \text { if } m=1, \forall n \\ \sum_{k=0}^{n} \beta_{m}(k) g(n-k, m-1), & \text { else }\end{cases}
$$

In the Eq. 5, $g(n, m)$ denotes the normalization constant of all possible sub closed queuing network where $n$ $(0 \leq n \leq N)$ and $m(1 \leq m \leq M)$ indicate, respectively, the amount of clients and nodes. Therefore, $g(N, M)$ represents $G(N)$.

\subsection{Marginal probability}

In order to compute the mean response time, it is necessary to evaluate the marginal probability. This performance measure $\pi_{m}(n)$ represents the probability of having $n$ clients in the node $m$, accordingly with Eq. 6 .

$$
\pi_{m}(n)=\sum_{\substack{\pi_{n_{1}} \ldots \pi_{n_{M}} \\ \& n_{m}=n}} \pi\left(n_{1}, \ldots, n_{M}\right)
$$

As mentioned, the number of states in a Markov chain of a complex system can become very huge. Therefore, it cannot be possible to compute all $\pi\left(n_{1}, \ldots, n_{M}\right)$. However, the convolution algorithm described in (Bolch et al., 1998) is sufficient to obtain the marginal probability directly. In general terms, this technique consist of to substitute Eq. 1 in Eq. 6, which results in Eq. 7.

$$
\pi_{m}(n)=\frac{\beta_{m}(n)}{G(N)} G_{M}^{(m)}(N-n)
$$

The constant $G_{M}^{(m)}(N-n)$ must be interpreted as normalization constant of a closed queuing network without the node $m$ and with $n$ clients less. The value of $G_{M}^{(m)}(N-n)$ can be computed recursively (Bolch et al., 1998), following Eq. 8.

$$
G_{M}^{(m)}(k)= \begin{cases}1, & \text { if } k=0 \\ G(N)-\sum_{k=1}^{N} \beta_{m}(n) G_{M}^{(m)}(N-k), & \text { else }\end{cases}
$$

\subsection{Mean response time}

As mentioned in section 1, the idea is to measure the mean response time of each node. Following the Little's law, this performance measure is obtained using Eq. 9:

$$
\bar{T}_{m}=\frac{\bar{N}_{m}}{\psi_{m}}
$$

where $\bar{N}_{m}$ and $\psi_{m}$ are the mean number of clients and the throughput of the node $m$, respectively. Moreover, these performance measures must be computed by Eq. 10 and Eq. 11, where $q_{m}$ denotes the number of services in the node $m$.

$$
\begin{gathered}
\bar{N}_{m}=\sum_{n=1}^{\infty} n \pi_{m}(n) \quad \forall 1 \leq m \leq M \\
\psi_{m}=\sum_{n=1}^{\infty} \pi_{m}(n) \min \left(n, q_{m}\right) \mu_{m}
\end{gathered}
$$

In this section, we show an efficient technique to compute the mean response time. This performance measure can be obtained analytically in a quiet fast. In the following section we introduce concepts about the load-haulage cycle and demonstrates how to use the mean response time to compute the total mine production index.

\section{Load-haulage cycle}

In (Torkamani and Askari-Nasab, 2012), a DES simulation model was implemented in SIMAN language (Pegden, 1983) in order to simulate the load-haulage system in an open-pit mine. Each simulation scenario used a distinct combination of the number of trucks and shovels. The goal was maximizing the mine production index at lowest possible operating cost. As the use of simulation requires high computational effort, sometimes it is not possible to try all feasible scenarios. Thus, the strategy taken by (Torkamani and Askari-Nasab, 2012) considers proper indicators to choose the scenarios to be evaluated.

In (Ercelebi and Bascetin, 2009), another strategy for allocation of trucks in open-pit mine was proposed. The authors applied closed queuing theory to obtain some measures of interest such as the total mine production index. According to (Ercelebi and Bascetin, 2009), in a load-haulage system, the total mine production index $P$ over a given time period of interest can be estimated by Eq. 12:

$$
P=N \cdot C \cdot \frac{T_{\text {horizon }}}{\bar{T}_{\text {cycle }}}
$$

where $C$ denotes the truck's capacity, $N$ the number of trucks and $T_{\text {horizon }}$ the period of interest. The measure $\bar{T}_{\text {Cycle }}$ represents the mean cycle time.

In a load-haulage system, basically, each truck goes to load site and waits until the loading process is completed. Following, the trucks go to the dump site and dump the ore into a crusher. However, each truck can be diverted to other process during the transport, such as maintenance, supply, etc. Known as operational stops, these processes must be included in the load-haulage cycle with a transient probability associated $p_{m}$. The Eq. 13 represents a general expression to compute the mean cycle time considering the operational stops:

$$
\bar{T}_{\text {cycle }}=\sum_{m=1}^{M^{\prime}} \bar{T}_{m}+\sum_{m=M^{\prime}+1}^{M} p_{m} \bar{T}_{m}
$$




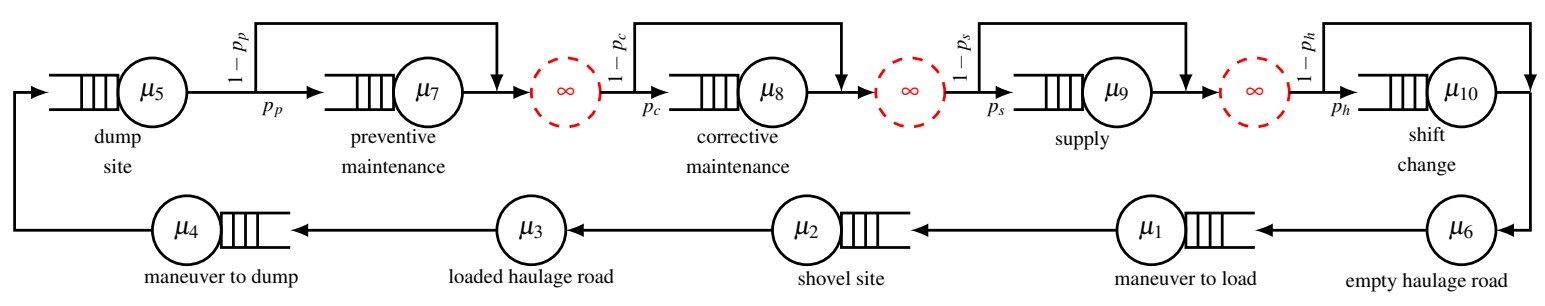

Figure 2. Closed queuing network representing the Load-haulage system

where the label $m$, from 1 to $M^{\prime}$ denotes the basic process of the load-haulage system, while $m$ from 1 to $M^{\prime}$ indicates process which are operational stops.

\section{DES model: Load-haulage system}

In order to compute the total mine production index, the analytical technique presented in this paper was tested in a load-haulage system depicting a mining front of a brazilian open-pit mine. As mentioned in section 1, in this study we consider the cdf approximation using exponential distribution. The assumption is verifying whether there are significant difference among the results obtained and the results presented in (Ribeiro, 2015). Table 1 presents the process of the load-haulage system. The pdf, the first moment approximation and discipline are showed as well.

Table 1. First moment approximation

\begin{tabular}{|c|l|l|l|l|}
\hline \hline Process & Dist. & $E[x]$ & $\mu_{m}$ & Dis \\
\hline $\begin{array}{c}\text { Maneuver } \\
\text { to load }\end{array}$ & Triangular & 2.266 & $\frac{1}{2.266}$ & FIFO \\
\hline $\begin{array}{c}\text { Shovel } \\
\text { site }\end{array}$ & $\begin{array}{c}\text { Tria } \\
\text { (comp.) }\end{array}$ & 3.227 & $\frac{1}{3.227}$ & FIFO \\
\hline $\begin{array}{c}\text { Loaded } \\
\text { Haulage road } \\
\text { Maneuver } \\
\text { to Dump } \\
\text { Dump } \\
\text { site }\end{array}$ & Gaussian & 8.333 & $\frac{1}{8.333}$ & IS \\
\hline $\begin{array}{c}\text { Empty } \\
\text { Haulage road }\end{array}$ & $\begin{array}{c}\text { Triangular. } \\
\text { Inv. }\end{array}$ & 1.100 & $\frac{1}{1.100}$ & FIFO \\
\hline $\begin{array}{c}\text { Preventive } \\
\text { maintenance }\end{array}$ & Triangular & 150 & $\frac{1}{2.133}$ & FIFO \\
\hline $\begin{array}{c}\text { Corrective } \\
\text { maintenance }\end{array}$ & Triangular & 1120 & $\frac{1}{150}$ & FIFO \\
\hline Supply & Gaussian & 150 & $\frac{1}{150}$ & FIFO \\
\hline $\begin{array}{c}\text { Shift } \\
\text { Change }\end{array}$ & Gaussian & 16 & $\frac{1}{16}$ & FIFO \\
\hline
\end{tabular}

The load-haulage system depicting a mining front was modeled as a queuing network. Thus, each process presented in Table 1 is seen as a node and each truck is a client. Figure 2 shows the full model. As we can see, there are four operational stops in this model. The transient probabilities to these nodes are: $p_{p}, p_{c}, p_{s}$ and $p_{h}$. In addiction, it was added to the model three fictitious nodes (dashed nodes) to establish transient decisions between operational stop nodes. Taken to account that these nodes have infinity rate, they do not change the final result.
The transient probabilities were computed using the occurrence period of each operational stop of the openpit mining front. Therefore, the estimated values are: $p_{p}=0.0015, p_{c}=0.0127, p_{s}=0.0255$ and $p_{h}=0.1232$.

Considering the transient probabilities values, the service rates and the queuing discipline showed in Table 1 the mean response time of each node is obtained using the technique presented in section 2. Consequently, using Eq. 13 we have the Eq. 14.

$$
T_{\text {cycle }}=\sum_{m=1}^{6} \bar{T}_{m}+p_{p} \bar{T}_{7}+p_{c} \bar{T}_{8}+p_{a} \bar{T}_{9}+p_{t} \bar{T}_{10}
$$

As mentioned before, the aim is to compute the total mine production index. However, it necessary to estimate the parameter $C$ (truck's capacity). In this study, it was considered a caterpillar 793F truck with nominal payload capacity of 226.8 tonnes. Finally, specifying a time period of interest, the mine production index must be evaluated hv Fo. 12.

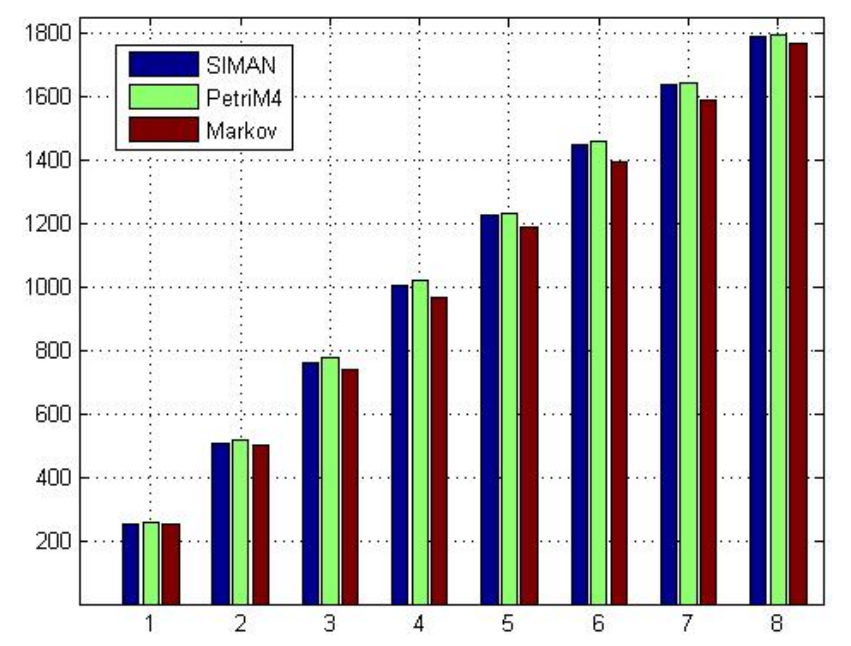

Figure 3. $N^{\mathrm{o}}$ of trucks vs Mine production index -tonnes(mil)

For a time period of interest of one month, the closed queuing network was evaluated changing the number of trucks $N$ (clients), from 1 to 8 . Figure 3 shows the comparison among the results obtained and the results presented in reference (Ribeiro, 2015).

From Figure 3 it is possible to observe there is no significant difference among the analytical approximation model designed and the methodologies of DES simulation, neither SIMAN nor Petri Nets. The maximum difference between the analytical approximation model and the 
SIMAN model was $4 \%$, while the maximum difference when compared with the Petri net model was 5.16\%.

It is important to emphasize that the analytical approximation model runs hundreds times faster than DES simulation models. In conclusion, the DES through markovian properties consist of a reasonable way to represent the load-haulage system in study.

\section{Project portfolio formulation}

Let $P_{0}$ be the total mine production index of the loadhaulage system shown in Figure 2, with 8 caterpillar 793F trucks and without any project application. Using Eq. 12 we have $P_{0}=1767.29$ tonnes. Let $P_{k}$ be the total mine production index of the same system with the application of the project $k$, and let $E\left[g_{k}\right]$ be the expected gain provided by the application of this project, that is $E\left[g_{k}\right]=P_{k}-P_{0}$.

Knapsack problem is a typical formulation for portfolio optimization. In this study, the goal consists of maximize the sum of the gains, respecting the established budget. However, it is necessary to consider the inter-relationship between two or more projects. Since it may be unfeasible to evaluated all project combinations, the mean response time of each node is useful to define those who deserve to be evaluated. The major idea is that this performance measure indicates the plant bottlenecks, which must be fixed by the new projects.

Initially, consider that two or more projects have a small expected gain. Using conventional optimization methods they would hardly be included in the final optimal portfolio. However, the combination of them can generate good expected gain when evaluated jointly. Considering these circumstances, the analysis of the problem is necessary to find which combinations are relevant.

For example, a project that produces a time reduction in the dumping process and increases mean response time in the shovel site can produce a significant gain whether combined with another projects that provides an improvement in the second node.

A relevant combination could be composed by more than two projects. In this methodology each combination just can be obtained by pairs. However, combinations found and evaluated are converted in new decision variables (new projects). Then, in the next iteration, the strategy can combine this new project with another one.

The Eq. 15 presents a formulation where the set of decision depends of the number of projects combination $\mathbf{S}=\left\{X_{0}, \ldots, X_{k}, \ldots, X_{K}\right\}$. Since $X_{k}$ denotes the $k$-th combination evaluated, $x_{k}$ is a decision variable that represents it in the optimization function.

Considering that in equation 15 the optimal solution can be composed by more than one project combination, it is necessary to prevent that a isolated project appear redundantly in the same solution. For example, suppose we have the combinations: $X_{1}=\left\{p_{1}, p_{2}\right\}$ and $X_{2}=\left\{p_{2}, p_{3}\right\}$. In this circumstances, it is necessary to append at the formulation (Eq. 15) the constraint $x_{1}+x_{2} \leq 1$. In an-
Table 2. Project portfolio

\begin{tabular}{|c|c|c|c|c|}
\hline Proj & Description & $c(\mathrm{pu})$ & Impact & Where? \\
\hline$p_{1}$ & $\begin{array}{l}\text { Bilateral } \\
\text { charging }\end{array}$ & 0.5 & $-15 \%$ & node 1 \\
\hline$p_{2}$ & $\begin{array}{c}\text { Roads } \\
\text { improvement }\end{array}$ & 1.5 & $-20 \%$ & nodes 3,6 \\
\hline$p_{3}$ & $\begin{array}{l}\text { Dead load } \\
\text { reduction }\end{array}$ & 1 & $5 \%$ & par. $C$ \\
\hline$p_{4}$ & Excavation & 2 & $-18 \%$ & node 2 \\
\hline$p_{5}$ & $\begin{array}{l}\text { 1 Truck } \\
\text { acquisition }\end{array}$ & 5 & $N+1$ & par. $N$ \\
\hline$p_{6}$ & $\begin{array}{l}2 \text { Truck } \\
\text { acquisition }\end{array}$ & 10 & $N+2$ & par. $N$ \\
\hline$p_{7}$ & $\begin{array}{l}\text { Rolling } \\
\text { A-Frame }\end{array}$ & 1 & $-17 \%$ & node 8 \\
\hline$p_{8}$ & $\begin{array}{c}\text { Forklif } \\
\text { acquisition }\end{array}$ & 0.7 & $-15 \%$ & node 8 \\
\hline$p_{9}$ & $\begin{array}{l}\text { Preventive } \\
\text { kits }\end{array}$ & 0.65 & $-14 \%$ & node 8 \\
\hline$p_{10}$ & $\begin{array}{c}\text { Supply } \\
\text { improvement }\end{array}$ & 2 & $-35 \%$ & node 9 \\
\hline$p_{11}$ & $\begin{array}{l}\text { Dump site } \\
\text { improvement }\end{array}$ & 2 & $-25 \%$ & node 4 \\
\hline$p_{12}$ & $\begin{array}{l}\text { Shift change } \\
\text { improvement }\end{array}$ & 1.5 & $-25 \%$ & node 10 \\
\hline$p_{13}$ & $\begin{array}{l}\text { Backlog's } \\
\text { reduction }\end{array}$ & 5 & $-30 \%$ & node 7 \\
\hline$p_{14}$ & $\begin{array}{l}\text { DMT } \\
\text { reduction }\end{array}$ & 2.5 & $-24 \%$ & node 5 \\
\hline$p_{15}$ & $\begin{array}{l}\text { Load site } \\
\text { improvement }\end{array}$ & 3 & $-25 \%$ & node 1 \\
\hline
\end{tabular}

other case, assume that we have two other project combination previously evaluated: $X_{3}=\left\{p_{3}, p_{4}\right\}$ and $X_{4}=$ $\left\{p_{1}, p_{2}, p_{3}, p_{4}\right\}$. Consequently, it is necessary to add the constraint $x_{1}+x_{3} \leq 1$ because a combination between this two set is equal to $X_{4}$. Based in this rules, the formulation must account the preposition 1:

Preposition 1 If $X_{u} \cap X_{v} \neq \emptyset$ or $X_{u} \cup X_{v}=X_{k} \mid k \in \mathbf{S}$ Then $x_{u}+x_{v} \leq 1$.

In this case, $X_{k}, X_{u}$ and $X_{v}$ are the combinations sets associated with the variables $x_{k}, x_{u}$ and $x_{v}$, respectively.

Let $w_{k}$ be the cost of the combination project $k$, the first constraint limits the sum of cost to the budget available $R$. Moreover, the last constraint indicates that the decision variables must be binary.

$$
\begin{array}{ll}
\text { Maximize } & \sum_{k=1}^{K} E\left[g_{k}\right] x_{k} \\
\text { Subject to } & \sum_{k=1}^{q} w_{k} x_{k} \leq R \\
& x_{u}+x_{v} \leq 1(\text { preposition } 1) \\
& x_{k}, x_{u}, x_{v} \in\{0,1\}
\end{array}
$$

For this study, it was available from the mine company a project portfolio with 15 candidate projects and a budget $R$ of $15 \mathrm{pu}$ (per unit). Table 2 presents the projects to be 
considered with the projects costs $(c)$. Moreover, Table 2 shows the impacts of the projects and the process (or parameter) affected.

This problem was formulated according to Eq. 15 with a limit of 200 'relevant combinations'. The 'Gurobi Optimizer' software was used and the best project portfolio solution was found. Lastly, the optimal solution was converted in a set of isolate project (such as Table 2) and the expected gain of this solution was obtained using the queuing network model presented in this paper. As a result, the best portfolio was $\{1,0,0,0,0,1,1,1,1,0,1,0,0,0,0\}$, which provides a production increase of 1049.09 tonnes.

\section{Conclusions}

In the context of projects portfolio, there is no simple way to select the best portfolio when considering the interrelationship between projects. Thus, this paper showed a strategy based on the characteristics of the load-haulage cycle of an open-pit mine, that limits cohesively the interrelationships to be evaluated, based on an adaptation of the knapsack problem. Given that the computational time is a limited resource, an analytical approximation model was designed. The results showed that there is not significant difference between this model and DES simulation models which represents the same load-haulage cycle.

\section{References}

Jerry Banks. Introduction to Simulation. Winter Sim. Conf., 2000.

Gunter Bolch, Stefan Greiner, Hermann de Meer, and Kishor S Trivedi. Queueing Networks and Markov Chains: Modeling and Performance evaluation with Computer Science applications. A Wiley-Interscience, 1998.

Jeffrey P Buzen. Computational Algorithms for Closed Queueing Networks with Exponential Servers. Com. of ACM, 16, 1973.

Christos G Cassandras. Introduction to Discrete event Systems. Springer Science Business Media, 2008.

Banu Y Ekren, Sunderesh S Heragu, Arvind Krishnamurthy, and Charles J Malmborg. An approximate solution for semi-open queueing network model of an autonomous vehicle storage and retrieval system. Automation Science and Engineering, IEEE Trans. on, 10(1):205-215, 2013.

S G Ercelebi and A Bascetin. Optimization of shovel-truck system for surface mining. The Journal of The Southern African Institute of Mining and Metallurgy, 109:433-439, 2009.

Michel C Fu. Optimization for Simulation: Theory vs. Practice. INFORMS Journal on Computing, 14:192-215, 2002.

W J Gordon and R R Newell. Closed Queueing Systems with Exponential Servers. Oper. Research, 15:254-265, 1967.

James R Jackson. Networks of waiting lines. Operations research, 5(4):518-521, 1957.
Raymond A Marie. Disappointments and Delights, Fears and Hopes induced by a few decades in Performance Evaluation. In Perf. Eval. of Comp. and Communication Systems. Milestones and Future Challenges, pages 1-9. Springer, 2011.

Barry L Nelson. Optimization via Simulation Over Discrete Decision Variables. Tuts. in Oper. Research - INFORMS, pages 193-207, 2010. doi:10.1287/educ.1100.0069.

C Dennis Pegden. Introduction to SIMAN. In Proc. of the 15th conf. on Winter simulation-Volume 1, pages 231-241. IEEE Press, 1983.

David Pisinger. A Minimal Algorithm for the Multiple-Choice Knapsack Problem. European Journal of Oper. Research, 83: 394-410, 1994. doi:10.1016/0377-2217(95)00015-I.

Cesar Monteiro Ribeiro. Modelagem e simulação de Sistemas a Eventos Discretos via redes de Petri estocásticas: Aplicação em mineração. Master's thesis, Universidade Federal de Minas Gerais, 2015.

Elmira Torkamani and Hooman Askari-Nasab. Verifying ShortTerm Production Schedules using Truck-Shovel Simulation. MOL Report Four, pages 302:1-16, 2012. 